\title{
Strain Tuning of the Anisotropy in the Optoelectronic Properties of $\mathrm{TiS}_{3}$
}

\author{
J. A. Silva-Guillén, ${ }^{*}, \dagger$ E. Canadell, F. Guinea, ${ }^{\ddagger}, \S$ and R. Roldán $\|$ \\ $†$ School of Physics and Technology, Wuhan University, Wuhan 430072, China \\ $\ddagger$ Fundación IMDEA Nanociencia, C/Faraday 9, Campus Cantoblanco, 28049 Madrid, \\ Spain \\ 【Institut de Ciència de Materials de Barcelona (ICMAB-CSIC), Campus Bellaterra, 08193 \\ Barcelona, Spain \\ $\S$ Department of Physics and Astronomy, University of Manchester, Oxford Road, \\ Manchester M13 9PL, UK \\ || Instituto de Ciencia de Materiales de Madrid, CSIC, Sor Juana Ines de la Cruz 3, 28049 \\ Cantoblanco, Madrid, Spain \\ E-mail: josilgui@gmail.com
}

\begin{abstract}
The benefits of two-dimensional (2D) materials for applications in nanotechnology can be widened by exploiting the intrinsic anisotropy of some of those crystals, being black phosphorus the most well known example. In this work we demonstrate that the anisotropy of $\mathrm{TiS}_{3}$, which is even stronger than that of black phosphorus, can be tuned by means of strain engineering. Using density functional theory calculations, we find that the ellipticity of the valence band can be inverted under moderate compressive strain, which is accompanied by an enhancement of the optical absorption. It is shown that the strain tuning of the band anisotropy can be exploited to focus plasmons in
\end{abstract}


the desired direction, a feature that could be used to design $\mathrm{TiS}_{3}$ nanostructures with switchable plasmon channeling.

\section{Keywords}

transition metal trichalcogenides, 2D materials, strain, anisotropy tuning, plasmons, optoelectronics.

The use of strain tuneable materials into optoelectronic devices makes possible to control optical and electronic properties by means of applied external strain. While such control has been shown to be feasible for several materials, most of them are electronically and optically isotropic in the propagation plane. ${ }^{1}$ However, new anisotropic $2 \mathrm{D}$ materials like black phosphorus, ${ }^{2-4}$ group V transition metal dichalcogenides $\mathrm{ReS}_{2}$ and $\mathrm{ReSe}_{2}{ }^{5-7}$ and transition metal trichalcogenide $\mathrm{TiS}_{3},{ }^{8}$ have been recently found to display strong linear dichroism. Furthermore, ultrathin materials of this type can be easily integrated into optoelectronic devices. Consequently, they may lead to novel functionalities based on the tuneability of their polarized light emission and/or absorption in devices for optical communication technologies. ${ }^{9}$

Two major developments have paved the way towards the emergence of such new generation devices. The advent of few-layer thick flakes of black phosphorous was an important step forward on this direction. ${ }^{10}$ Thin flakes of this material possess band gaps of the suitable order of magnitude and good tuneability, as well as high mobilities to be integrated in devices for photovoltaic, thermal imaging and telecommunication applications. ${ }^{2}$ However, what makes really attractive black phosphorous is its unusual in-plane anisotropy. ${ }^{3,11}$ Its definite linear dichroism really distinguishes black phosphorous from graphene, molybdenum and tungsten dichalcogenides or other 2D materials whose electronic structure is markedly isotropic ${ }^{12}$ thus opening the way to a whole new range of potential applications. ${ }^{13}$

Another important step forward on this direction was achieved with the preparation of 

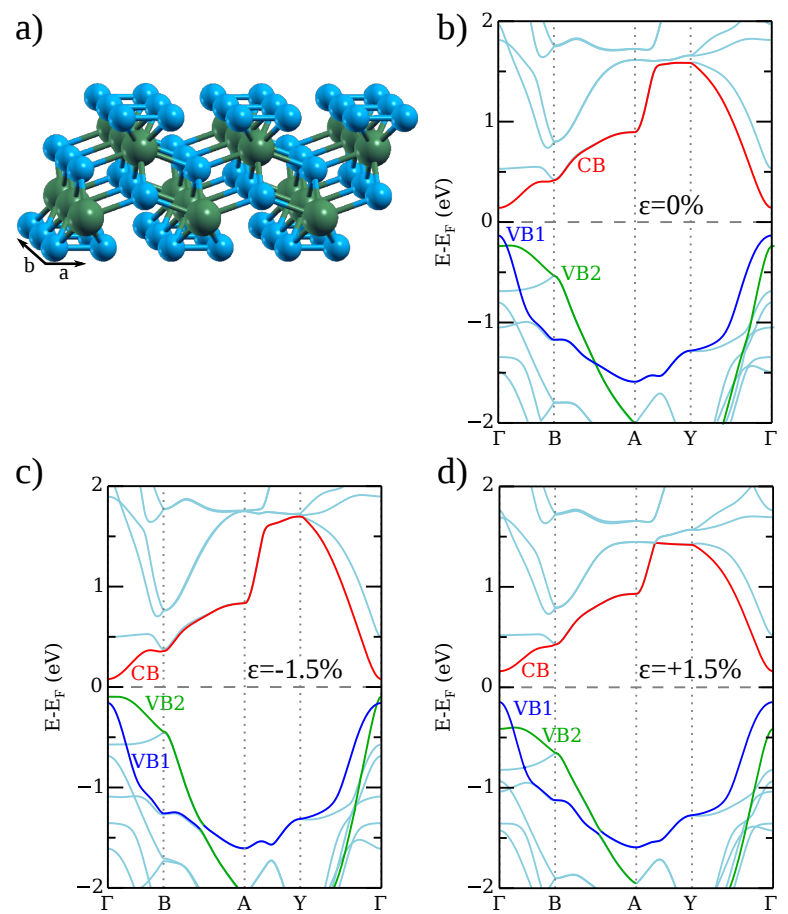

Figure 1: (a) Crystal structure of $\mathrm{TiS}_{3}$, where green and blue correspond to $\mathrm{Ti}$ and $\mathrm{S}$ atoms, respectively. Calculated band structure for single-layer $\mathrm{TiS}_{3}$ for the optimized structure (b), for a compressive strain of $-1.5 \%$ (c) and for a tensile strain of $+1.5 \%(\mathrm{~d})$. $\Gamma=(0,0,0), \mathrm{B}=$ $(1 / 2,0,0), \mathrm{A}=(1 / 2,1 / 2,0), \mathrm{Y}=(0,1 / 2,0)$ in units of the reciprocal lattice vectors. The Fermi level is set at zero. Highlighted in red, green and blue are the three bands of interest, the conduction band (CB) and the two valence bands (VB1 and VB2), respectively.

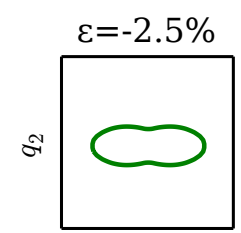

$q_{1}$

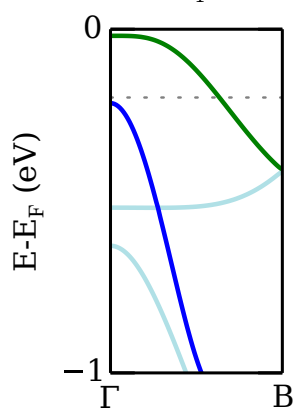

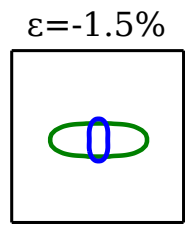

$q_{1}$

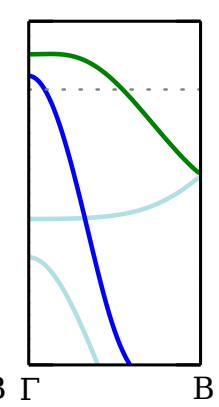

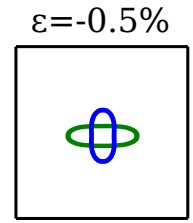

$q_{1}$

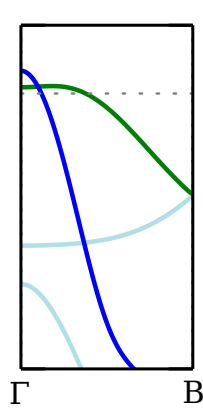

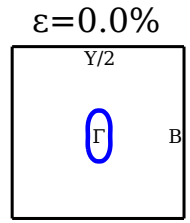

$q_{1}$

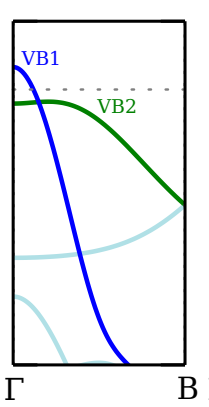

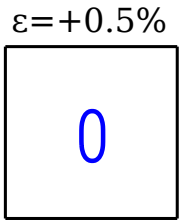

$q_{1}$

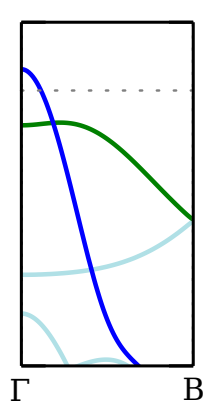

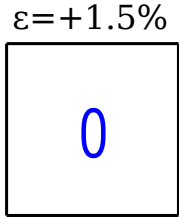

$q_{1}$

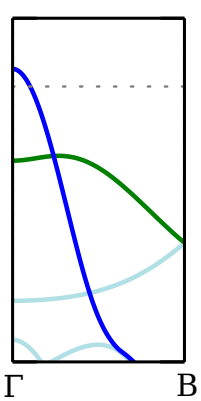

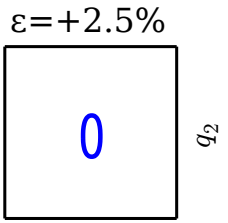

$q_{1}$

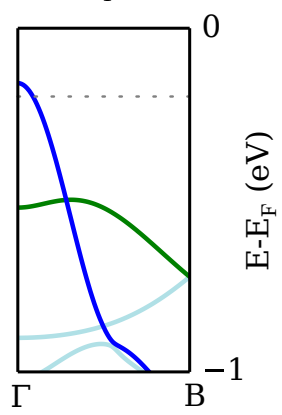

Figure 2: Strain driven band inversion in single layer $\mathrm{TiS}_{3}$. Evolution of the valence bands (bottom panel) with their corresponding constant energy contours evaluated for the energy associated with the dashed line (top panel). Note that the top of the vertical coordinate in the top panel lies at $\mathrm{Y} / 2$. 
thick flakes of group IV transition metal trichalcogenide $\mathrm{TiS}_{3}$ and other members of this family. ${ }^{14-17}$ Atomically thin $\mathrm{TiS}_{3}$ is an anisotropic semiconductor with opposite ellipticity in the valence and conduction bands. ${ }^{15,18-22}$ Thus, in that case the possibility arises to reverse the anisotropy of the electrical and optical properties by switching from $n$ - to $p$-doping or viceversa. ${ }^{23}$ Angle-resolved photoluminiscence measurements for another member of this family, $\mathrm{ZrS}_{3}$, have proved that few-layer flakes of this material indeed exhibit very anisotropic optical properties. ${ }^{24}$ Recent theoretical work has shown that tensile strain may lead to the enhancement of the carrier mobility of $\mathrm{TiS}_{3}$ single-layers by one order of magnitude ${ }^{25}$ and that the Poisson ratio and in-plane stiffness are highly anisotropic. ${ }^{26}$ Unusual vibrational properties with a large in-plane anisotropy have also been reported for $\mathrm{TiS}_{3}$ whiskers ${ }^{27}$ and angle resolved Raman studies for mechanically exfoliated $\mathrm{TiS}_{3}, \mathrm{ZrS}_{3}$ and $\mathrm{HfS}_{3}$ crystals have shown that the intensity of several Raman modes exhibits a strong polarization dependence. ${ }^{28}$

The electronic structure of semiconductors of the $\mathrm{TiS}_{3}$ family still exhibits an additional appealing feature: there are two close bands with different character and anisotropy at the top of the valence band. Using first-principles calculations we have found that the order of these two bands may be inverted in $\mathrm{TiS}_{3}$ single-layers upon compression. This feature opens the additional possibility of reversing the anisotropy of the valence band by strain engineering. Materials of the $\mathrm{TiS}_{3}$ family thus rank among the most versatile for optoelectronic applications and its strain engineering certainly deserves detailed attention.

Here we report a first-principles DFT study of single-layers of $\mathrm{TiS}_{3}$ showing that the opposite directional anisotropy of the valence and conduction bands is kept under variations in the tensile strain whereas the anisotropy of the valence band can be changed by a slight compressive strain. Thus, single-layer $\mathrm{TiS}_{3}$ is predicted to be a material supporting highly anisotropic transport, excitons and plasmons for the conduction and valence bands that can be tuned to be of either opposite or equal anisotropy. The strong light-matter interaction that occurs in plasmonic materials allows for light confinement and electric-field localisation at sub-wavelength scales. Graphene is the $2 \mathrm{D}$ material that has been most widely studied as an 
atomically thin plasmonic crystal in the infrared and terahertz optical range. ${ }^{29}$ However, the optoelectronic properties of graphene are isotropic. ${ }^{30}$ The strain tuning of the band ellipticity that we have found opens the possibility to use $\mathrm{TiS}_{3}$ for directional dependent plasmonic devices, ${ }^{31}$ polarization sensors, ${ }^{32}$ hyperbolic plasmons, ${ }^{33}$ focusing of strain induced exciton funnels ${ }^{34}$ or anisotropic in-plane thermoelectric applications, ${ }^{35}$ among others.

A single layer of $\mathrm{TiS}_{3}$ is formed from trigonal prismatic $\mathrm{TiS}_{3}$ chains in such a way that two rectangular faces of an $\mathrm{TiS}_{6}$ trigonal prism are capped by $\mathrm{S}$ atoms of the neighboring chains (see Fig. 1a). Thus, every Ti atom is coordinated to eight chalcogen atoms, S. The calculated band structure for the optimized structure of the single-layer $\mathrm{TiS}_{3}$ is shown in Fig. 1b. The obtained anisotropy of the valence band is in good agreement with recent ARPES measurements. ${ }^{36}$ With the PBE functional we find a direct gap of $246 \mathrm{meV}$ at $\Gamma$. This is in good agreement with previous plane wave-based DFT calculations. ${ }^{19-21,37}$ As noted previously ${ }^{19,23}$ the PBE functional underestimates the band gap by $\sim 0.7 \mathrm{eV}$. However, calculations using the HSE06 functional, ${ }^{38}$ which leads to an appropriate value of $1.12 \mathrm{eV}$, show that the band structures calculated with the two functionals are practically identical except for an almost rigid shift of the empty bands. Consequently, from now on our analysis will be based on the PBE type calculations which, in all the cases studied, keep the band gap. Our results show that compressive strain (Fig. 1c) decreases the band gap whereas tensile strain (Fig. 1d) increases it. In agreement with these results, it has been experimentally observed that tensile strain induces an increase of up to $10 \%$ in the band gap. ${ }^{39}$ It is clear from Fig. 1 that the effect of strain is stronger for the valence bands. Remarkably, upon a small compressive strain there is a change in the nature of the band occurring at the top of the valence bands, where two bands of different orbital origin and opposite anisotropy are inverted (see Fig. 2). The strain driven change of ellipticity is clearly seen in the constant energy contours shown in the top panels of Fig. 2. Judging from the different dispersion of these two bands along the main directions of the single-layer, this inversion could have important consequences for the plasmons anisotropy, as we will discuss later. 

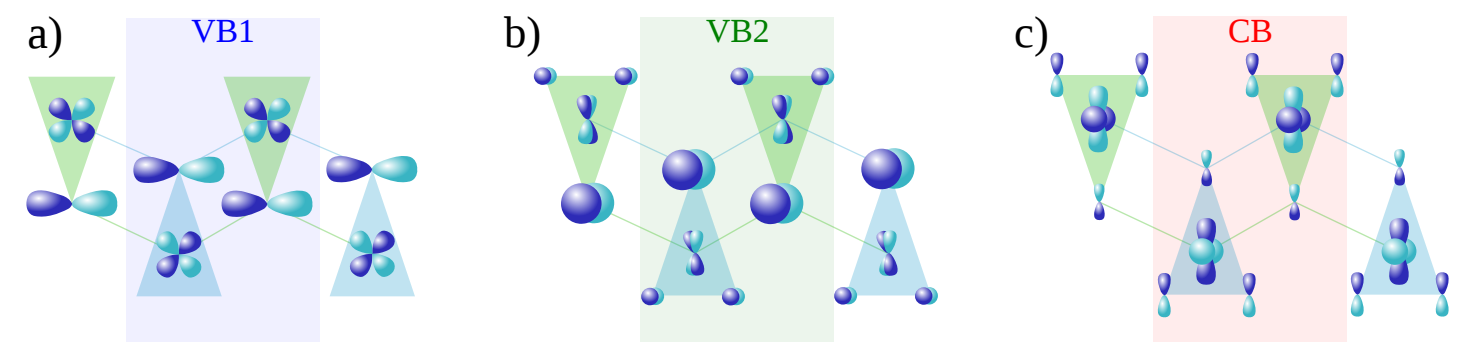

Figure 3: Schematic representation of the upper valence band VB1 (a), the second valence band VB2 (b) and the lowest conduction band CB (c) crystal orbitals at $\Gamma$ in the unstrained structure of single-layer $\mathrm{TiS}_{3}$. The triangles shown in green and blue are displaced by $b / 2$ so that all atom-atom distances with the same color occur in the same plane. The filled rectangular area is the projection of the unit cell and the smaller side is the $a$ parameter.

In order to clearly grasp the origin of these results, we remind here some relevant details of the crystal and electronic structure of $\mathrm{TiS}_{3}$ (discussed in more detail in Ref. 23): (i) The top of the valence band VB1 is mostly made of the S $3 p_{x}$ orbitals and a smaller contribution of the Ti $3 d_{x y}$ orbitals (Fig. 3a). The S $3 p_{x}$ contribution comes only from the inner sulfur atoms of the triangles (i.e. those not forming the short S-S bonds, see Fig. 1a). Consequently, the upper valence band concentrates in the inner part of the layer and is mostly directed along the a-direction. (ii) Very near the top of the valence band it occurs a second band VB2 whose main orbital contribution comes from S $3 p_{z}$ orbitals of both inner and outer sulphur atoms of the triangles (Fig. 3b). (iii) The bottom of the conduction band is mainly based on the Ti $3 d_{z^{2}}$ orbitals mixed with Ti $3 d_{x^{2}-y^{2}}$ leading to a Ti $3 d_{z^{2}-y^{2}}$ (see Fig. $3 \mathrm{c}$ and detailed discussion in Ref. 23) strongly pointing along one of the two directions of the layer, $b$, what leads to a strong anisotropy to the electron carriers.

In view of these observations it is easy to understand why the two upper valence bands at $\Gamma$ have different anisotropies. Since it is strongly based on the S $3 p_{x}$ orbitals of the inner non bonded S atoms, the upper one VB1 is going to have larger dispersion and thus, smaller effective mass, along the interchain $a$-direction. In contrast, because the second upper VB2 band is mainly based on the S $3 p_{z}$ orbitals of both inner and outer sulphur atoms, the effective mass will be smaller along the chain $b$ direction. It is thus challenging to try to understand if it is possible to reverse the order of these two bands and thus, the directional 
anisotropy of the valence band. Let us first consider the second valence band VB2. Around $\Gamma$ the $\mathrm{S} 3 p_{z}$ orbitals undergo strongly antibonding interactions with those of the $\mathrm{S}$ atoms above and below because they point directly to each other and they have the same sign. Consequently, compression along $b$ considerably destabilizes the top of the band whereas compression along $a$ does not have a strong effect. Thus, biaxial compression destabilizes this band. Of course, stretching has the opposite effect.

Let us now consider the upper valence band VB1. As mentioned above, because of the $b / 2$ offset of the successive trigonal prismatic chains, the Ti atom of one of the chains is capped by two $\mathrm{S}$ atoms of the two adjacent chains. Consequently, the $\mathrm{Ti}$ atoms make six bonds with the $\mathrm{S}$ atoms of its own chain and two bonds with $\mathrm{S}$ atoms of the adjacent chains. The nature of the crystal orbital at $\Gamma$ is schematically shown in Fig. 3a. Although the dominant character is $\mathrm{S} 3 p_{x}$ of the inner $\mathrm{S}$ atoms, there is a definite participation of the Ti $3 d_{x y}$ orbitals which mix in a bonding way. When compressing or stretching the layers along the interchain direction, $a$, the main structural change is a variation of the $\mathrm{S}-\mathrm{Ti}-\mathrm{S}$ angle associated with the capping $\mathrm{S}$ atoms. This leads to two competing effects. The interaction between S $3 p_{x}$ orbitals of adjacent chains changes because both the distance and the orbital orientation are modified. In addition, since the $\mathrm{S}-\mathrm{Ti}-\mathrm{S}$ angle changes, the interaction between the $\mathrm{Ti} 3 d_{x y}$ and S $3 p_{x}$ orbitals also change. When stretching, the S-S distance becomes larger and the orbital orientation is energetically less favourable so that both factors favour a stabilization of this band since the S-S interaction is antibonding. Under compression the effect is just the opposite. As far as the interaction between the Ti $3 d_{x y}$ and S $3 p_{x}$ orbitals is concerned, given the value of the $\mathrm{S}-\mathrm{Ti}-\mathrm{S}$ angle ( 140 degrees), compression will lead to a decrease of this angle, which will increase the overlap and thus will stabilize the orbital. Stretching leads to an increase of the angle, which will thus contribute to the destabilization of this orbital. Thus, both for compressive and tensile strain, the two factors are in competition and this is why the variation of VB1 is considerably weaker than it is for the lower valence band VB2. Only for relatively large values of the strain this level exhibits a sizable variation and in both 
cases it is slightly stabilized.

In summary, whereas the upper valence band VB1 exhibits a rather soft variation with strain, the second one VB2 considerably raises under compression. It follows that there is no way to reverse the order of the two valence bands under tensile strain. However, since in the unstrained structure the two bands are very near each other, it is clear that a very small compression should be able to reach this goal. According to our calculations, the crossing should occur for values as weak as a $1 \%$ compression. Considering that the DFT optimized structure for bulk $\mathrm{TiS}_{3}$ is slightly larger than the experimental one, ${ }^{19}$ we expect that this $1 \%$ is an overestimation, and that the true transition might take place for smaller values of compressive strain, well within the experimentally reachable strains in the laboratory.

Finally, let us consider the evolution of the bottom conduction band CB under strain. Since this level is strongly based on the Ti $3 d_{z^{2}-y^{2}}$ orbitals (Fig. 3c) it undergoes strong interactions along the intrachain $b$ direction. At $\Gamma$, these interactions are bonding along the $\mathrm{Ti}-\mathrm{Ti}$ direction $b$, so that when the $\mathrm{Ti}-\mathrm{Ti}$ distances decrease/increase the crystal orbital is stabilized/destabilized. The variations brought about by changes in the interchain, $a$, direction are quite weak compared with those along the chain because of the good overlap of the Ti $3 d_{z^{2}-y^{2}}$ orbitals along $b$ which overrides all other effects for the range of strains considered here. Thus we conclude that under tensile strain the band gap of single-layer $\mathrm{TiS}_{3}$ increases, as experimentally found, ${ }^{39}$ and it must decrease under compressive strain.

Before exploring more carefully the consequences of these variations with either compressive or tensile strain, let us summarize the main results concerning the variation of the band gap and effective masses. In Fig. 4a, we show the difference in energy of the three bands of interest (CB, VB1 and VB2). Compression of the sample brings together VB1 and VB2 and eventually, and inversion of those bands occurs for about $1 \%$ of strain, VB2 becoming the effective valence band (Fig. 2). If we apply a tensile strain, VB1 and VB2 move apart. Since most of the transport properties of a semiconductor depend on the effective masses of the carriers, it is interesting to analyze how they change with the application of an external 

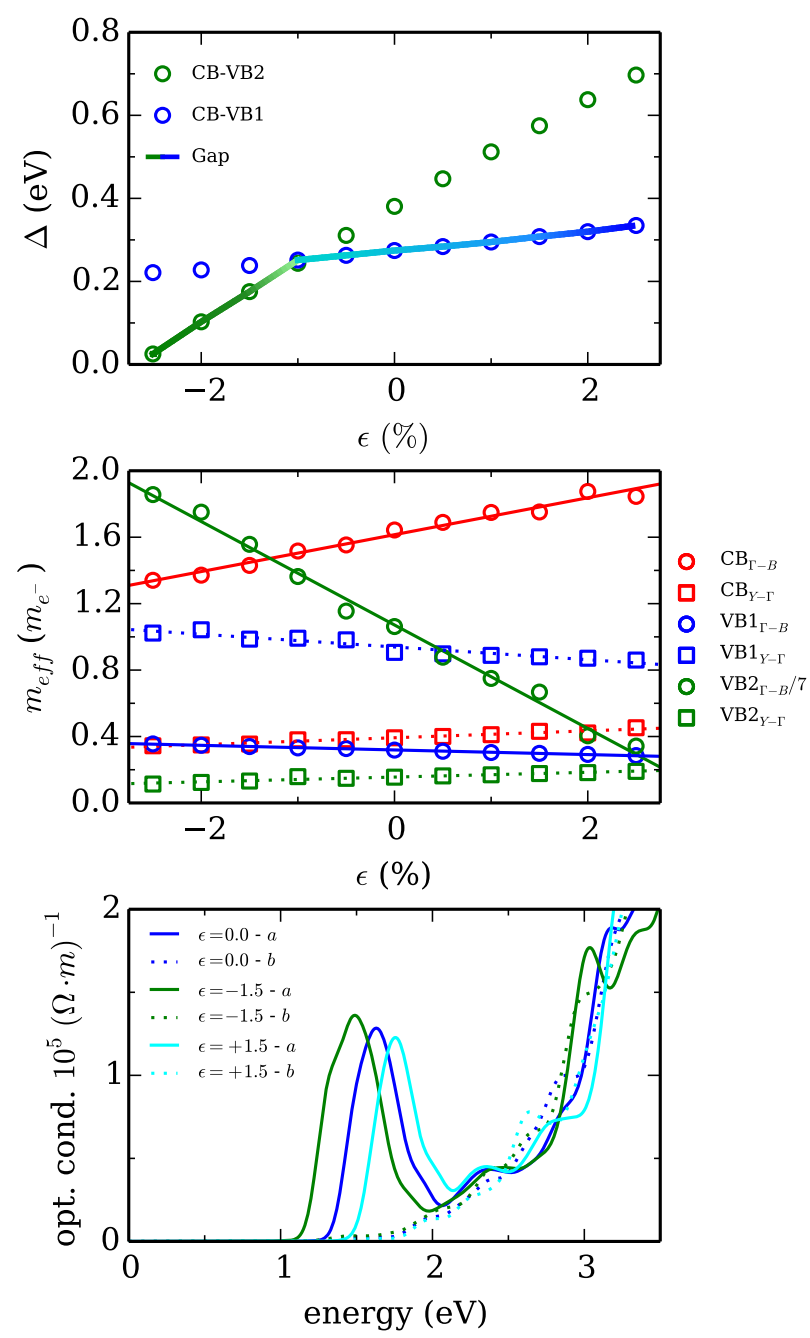

Figure 4: (a) Change of the bandgap with strain. Different color correspond to gap between the conduction band and the two upper valence bands for different values of strain, and the solid line indicates the minimal gap. (b) Effective mass anisotropy and its dependence with applied strain. Notice a factor of $1 / 7$ in the $\mathrm{VB} 2_{\Gamma-B}$ curve. (c) Optical conductivity for different strains along the $a$ (solid line) and $b$ (dotted line) directions (the energy has been shifted $+0.7 \mathrm{eV}$ to match the corrected gap value). 
strain. The results are given in Fig. 4b. Although for most of the bands the change is small, it is important to note that for VB2 this is not the case. This is due to the fact that VB2 is a weakly dispersing, flat band that bends when strain is applied, leading to substantial modification the effective masses. Other possible routes to tune the anisotropy and the effective masses in $\mathrm{TiS}_{3}$ include the application external out-of-plane electric field, or controlling the stacking angle in multilayer samples, as it has been recently demonstrated for black phosphorus. ${ }^{40}$
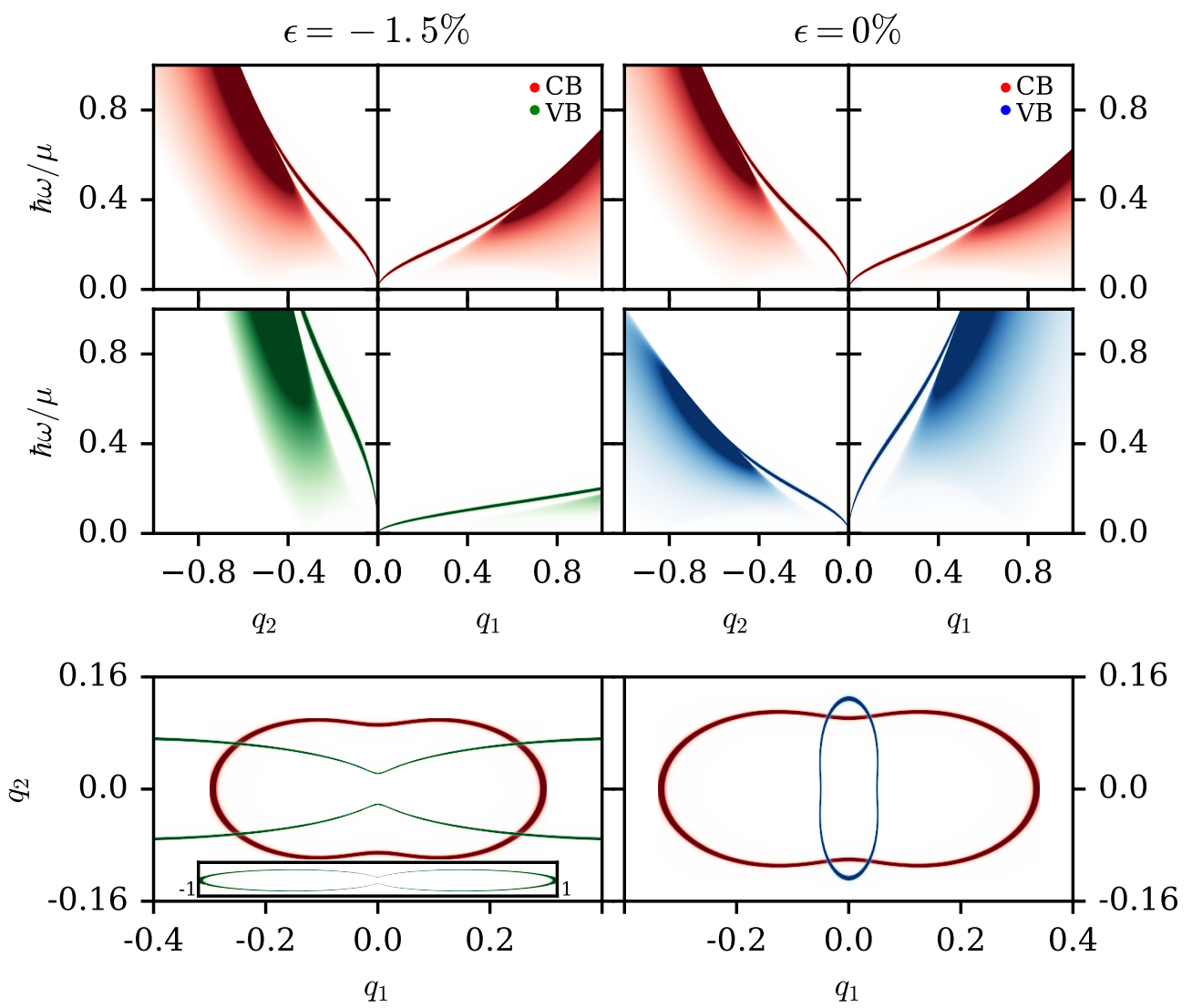

Figure 5: Top: Plasmon spectrum of compressed and unstrained $\mathrm{TiS}_{3}$. Bottom: corresponding cross section of the loss function that show the plasmon dispersion for the valence and conduction bands. The inset in the bottom left panel shows the full contribution of the VB2 band. The calculations have been done for a carrier density of $3 \cdot 10^{13} \mathrm{~cm}^{-2}$ in the case of the conduction band and $5 \cdot 10^{13} \mathrm{~cm}^{-2}$ for the valence band.

We have also studied the optical conductivity $\sigma_{i j}(\omega)$ of both unstrained and strained single layer $\mathrm{TiS}_{3}$, where $i, j=a, b$. In agreement with previous experimental results for 
unstrained $\mathrm{TiS}_{3},{ }^{41}$ the system presents a large linear dichroism, that is, a strong difference in optical conductivity for incident light polarized along $a$ and $b$ directions (see Fig. 4c). In fact, for polarization along the $a$ direction, the band edge of the first absorption peak occurs at the bandgap energy and then decreases considerably. On the other hand, the spectrum for b-polarized light shows a much weaker response at low energies. This suggests that, as in the case in black phosphorus, ${ }^{11}$ the symmetries of the wave-functions lead to a dipole operator that connects the valence and conduction band states for $a$-polarization, allowing for direct inter-band transitions, which are symmetry-forbidden for $b$-polarization. Furthermore, the tuning of bandgap with tensile (compressive) strain leads to a blue (red) shift of the onset of optical inter-band transitions at photon energy equal to the corresponding gap. Importantly, the intensity of $\sigma_{a a}$ increases with compressive strain (see also Fig. S2 of Supp. Info. for polar plots of $\sigma_{i j}(\omega)$ ). This is due to the compression driven valence band inversion which, as previously discussed, leads to same ellipticity in valence and conduction bands, with the corresponding enhancement of allowed optical inter-band transitions. We further mention that the anisotropy of the electronic properties of $\mathrm{TiS}_{3}$ can lead to unbalance between the electrical and thermal properties, making this material also promising for thermoelectric applications. $^{42}$

We finally discuss the consequences of strain for the collective excitation spectrum of $\mathrm{TiS}_{3}$. Plasmons in 2D materials are being extensively studied for applications in nanoplasmonics and optoelectronics. They are highly confined, due to their atomically thin nature, and can be controlled by different means, like external gate or chemical doping. ${ }^{30,43}$ Plasmon modes in single-layer $\mathrm{TiS}_{3}$ were shown to be anisotropic with opposite anisotropy for the valence and conduction bands. ${ }^{23}$ In the following we show that the dispersion relation and anisotropy of plasmons in $\mathrm{TiS}_{3}$ can be tuned by application of strain. For this we calculate the loss function $\mathcal{L}(\boldsymbol{q}, \omega)$ within the Random Phase Approximation (RPA) (see Methods). Our results for the interacting single-particle spectrum in the frequency-momentum plane are shown in Fig. 5 (top panels). For the cases of relaxed $(\varepsilon=0 \%)$ and compressed $(\varepsilon=-1.5 \%)$ 
crystals, we have calculated the plasmon modes originating from the collective oscillation of carriers in the $\mathrm{CB}$ or in the VB (electron or hole doping, respectively). As expected, the results for the $\mathrm{CB}$ (red color) are similar for $\varepsilon=0 \%$ and $\varepsilon=-1.5 \%$, due to the weak effect of strain in the conduction band. On the other hand, the plasmon dispersion for hole doping (VB) dramatically changes from the unstrained to the compressed cases. It is clear that the plasmon velocity (as given by the slope of the plasmon branch) for the $\varepsilon=0 \%$ (blue color) is higher for $\mathbf{q} \| \mathbf{q}_{\mathbf{1}}$, whereas the opposite happens for $\varepsilon=-1.5 \%$ (green color), for which the plasmon mode is faster for momenta $\mathbf{q} \| \mathbf{q}_{2}$. This effect is more clearly seen in the cross section plots of the corresponding loss functions, given in the bottom panels of Fig. 5: while the anisotropy of the plasmon modes is opposite for $\mathrm{CB}$ and VB if $\varepsilon=0 \%$ (inverted ellipticities), this is not the case for the compressed case, for which the ellipticity of the cross sections is the same. The above results make evident that a slight compressive strain may completely change the anisotropy of the hole plasmon branch, due to the inversion of the valence bands VB1 and VB2 with opposite ellipticity. This opens the possibility to focus the flow of plasmons, tuning the direction of propagation by means of external strain.

In summary, our first-principles DFT study of biaxial strain on single-layers of $\mathrm{TiS}_{3}$ show that the band gap increases/decreases under tensile/compressive strain. While the opposite anisotropy of valence and conduction bands remains under application of tensile strain, we have demonstrated that moderate compressive strain leads to an inversion of the valence bands accompanied by a change of the ellipticity. This makes that valence and conduction bands present the same or opposite anisotropies depending on the applied external compression. We have shown the consequences of such effect on the optical properties, finding an enhancement of the optical conductivity due to activation of inter-band optical transitions when the band inversion takes place. Furthermore, we have demonstrated that strain tuning of the band anisotropy can be exploited to focus plasmons in the desired direction, with potential applications for optoelectronic nanodevices. Since the compression needed to reach the transition $(\sim 1 \%)$ is well within the experimentally available range, we hope our 
results will motivate future studies to confirm that strain engineering can be used to tune the anisotropy of $\mathrm{TiS}_{3}$.

Methods. First-principles calculations were carried out using a numerical atomic orbitals approach to DFT, ${ }^{44,45}$ which was developed for efficient calculations in large systems and implemented in the SIESTA code. ${ }^{46,47}$ We have used the generalized gradient approximation (GGA) and, in particular, the functional of Perdew, Burke and Ernzerhof. ${ }^{48}$ Only the valence electrons are considered in the calculation, with the core being replaced by norm-conserving scalar relativistic pseudopotentials ${ }^{49}$ factorized in the Kleinman-Bylander form. ${ }^{50}$ The non-linear core-valence exchange-correlation scheme ${ }^{51}$ was used for all elements. We have used a split-valence double- $\zeta$ basis set including polarization functions. ${ }^{52}$ The energy cutoff of the real space integration mesh was set to $500 \mathrm{Ry}$. To build the charge density (and, from this, obtain the DFT total energy and atomic forces), the Brillouin zone (BZ) was sampled with the Monkhorst-Pack scheme ${ }^{53}$ using grids of $(30 \times 30 \times 1) k$-points. The biaxial strain was applied in both the $a$ and $b$ directions (see Fig.1). The atomic positions were relaxed after applying the strains to the single-layer. The strain is defined as $s=\delta m / m_{0}$ where $m_{0}$ is the unstrained cell parameter and $\delta m+m_{0}$ the strained cell parameter. Thus, positive values correspond to tensile strain whereas negative numbers correspond to compressive strain. The computation of the optical properties is performed calculating the dipolar transition matrix elements of different eigenfunctions of the Hamiltonian, as implemented in the SiESTA code.

The plasmon spectrum was obtained from the zeros of the dynamical dielectric function $\varepsilon(\boldsymbol{q}, \omega)$, calculated within the $\operatorname{RPA}^{54,55} \varepsilon(\boldsymbol{q}, \omega)=1-v(\boldsymbol{q}) \Pi^{0}(\boldsymbol{q}, \omega)$, where Coulomb interaction is $v(\boldsymbol{q})=\frac{2 \pi e^{2}}{\kappa q}$ where $\kappa$ is the background dielectric constant and $\Pi^{0}(\mathbf{q}, \omega)$ is the polarization function

$$
\Pi^{0}(\boldsymbol{q}, \omega)=\frac{g_{s}}{\mathcal{V}} \sum_{\boldsymbol{k}} \frac{f_{F}(\boldsymbol{k})-f_{F}(\boldsymbol{k}+\boldsymbol{q})}{\xi(\boldsymbol{k}+\boldsymbol{q})-\xi(\boldsymbol{k})-\hbar(\omega+i \delta)}
$$


where $\mathcal{V}$ is the system size, $g_{s}=2$ is the spin degeneracy, $f_{F}(\boldsymbol{k})=\left\{\exp \left[\xi(\boldsymbol{k}) / k_{B} T\right]+1\right\}^{-1}$ is the Fermi-Dirac distribution function, and $\delta$ is a phenomenological broadening. The energy dispersion within the effective mass approximation is $\xi^{c, v}(\boldsymbol{k})=\frac{\hbar^{2} k_{1}^{2}}{2 m_{1}^{c, v}}+\frac{\hbar^{2} k_{2}^{2}}{2 m_{2}^{c, v}}-\mu$ where the superscript $c(v)$ indicates conduction (valence) band, $\mu$ is the chemical potential, and the corresponding effective masses have been obtained from the DFT calculations of strained $\mathrm{TiS}_{3}$ (Fig. 4b). The value of $\mu$ was set to $0.1 \mathrm{eV}$ in the case of $\epsilon=-1.5 \%$ for both conduction and valence band and to $0.09 \mathrm{eV}$ and $0.22 \mathrm{eV}$ in the case of $\epsilon=0.0 \%$ for the conduction and valence band, respectively. This is equivalent to a carrier density of $3 \cdot 10^{13}$ $\mathrm{cm}^{-2}$ in the case of the conduction band and $5 \cdot 10^{13} \mathrm{~cm}^{-2}$ in the valence band (it is worth noticing that we use different values for $\mu$ in order to have the same valence and conduction carrier densities in the different calculations). The anisotropy is included in the polarization function following Ref. ${ }^{23}$

\section{Supporting Information Available}

Supporting information includes the evolution of the band structure with strain, ranging from $+7.5 \%$ (tensile) to $-1.5 \%$ (compresive) and the effective masses for the three bands of interest of the $\mathrm{TiS}_{3}$ single-layer.

\section{Acknowledgments}

This work has received funding from the European Unions Seventh Framework Programme (FP7/2007-2013) through the ERC Advanced Grant NOVGRAPHENE (GA No. 290846), European Commission under the Graphene Flagship, contract CNECTICT-604391, the Spanish MINECO (through Grants No. FIS2015-64886-C5-4-P, FIS2014-58445-JIN, Ramón y Cajal program RYC-2016-20663, and the Severo Ochoa Centers of Excellence Program under Grant SEV-2015-0496), and the Generalitat de Catalunya (2017SGR1506). 


\section{References}

1. Manzeli, S.; Ovchinnikov, D.; Pasquier, D.; Yazyev, O.; Kis, A. 2D transition metal dichalcogenides. Nat. Rev. Mater. 2017, 2, 17033.

2. Castellanos-Gomez, A. Black Phosphorous: Narrow Gap, Wide Applications. J. Phys. Chem. Lett. 2015, 6, 4280-4291.

3. Ling, X.; Wang, H.; Huang, S.; Xia, F.; Dresselhaus, M. S. The renaissance of black phosphorous. Proc. Natl. Acad. Sci. 2015, 112, 4523-4530.

4. Fei, R.; Yang, L. Strain-Engineering the Anisotropic Electrical Conductance of FewLayer Black Phosphorous. Nano Lett 2014, 14, 2884-2889.

5. Yang, S.; Wang, C.; Dahin, H.; Chen, H.; Li, Y.; Li, S.-S.; Suslu, A.; Peeters, F. M.; Liu, Q.; Li, J. et al. Tuning the Optical, Magnetic, and Electrical Properties of ReSe 2 by Nanoscale Strain Engineering. Nano Lett. 2015, 15, 1660-1666.

6. Lin, Y.-C.; Kosma, H.-P.; Yeh, C.-H.; Björkman, T.; Liang, Z.-Y.; Ho, C.-H.; Huang, Y.S.; Chiu, P.-W.; Krasheninnikov, A. V.; Suenaga, K. Single-Layer ReS ${ }_{2}$ : TwoDimensional Semiconductor with Tunable In-Plane Anisotropy. ACS Nano 2015, 11, $11249-11257$.

7. Chenet, D.; Burak Aslan, O.; Huang, P. Y.; Fan, C.; van der Zande, A. M.; Heinz, T. F.; Hone, J. C. In-Plane Anisotropy in Mono- and Few-Layer $\mathrm{ReS}_{2}$ Probed by Raman Spectroscopy and Scanning Transmission Electron Microscopy. Nano Lett. 2015, 15, 56675672 .

8. Island, J. O.; Molina-Mendoza, A. J.; Barawi, M.; Biele, R.; Flores, E.; Clamagirand, J. M.; Ares, J. R.; Sánchez, C.; van der Zant, H. S. J.; D’Agosta, R. et al. Electronics and optoelectronics of quasi-one dimensional layered transition metal trichalcogenides. 2D Materials 2015, 4, 022003. 
9. Martella, C.; Mennucci, C.; Lamperti, A.; Cappelluti, E.; de Mongeot, F. B.; Molle, A. Designer Shape Anisotropy on Transition Metal Dichalcogenide Nanosheets. Advanced Materials 2018, 30, 1705615.

10. Lin, L.; Yu, Y.; Ye, G. J.; Ge, Q.; Ou, X.; Wu, H.; Feng, D.; Chen, X. H.; Zhang, Y. Black Phosphorous Field-Effect Transistors. Nat. Nanotechnol. 2014, 9, 372-377.

11. Xia, F.; Wang, H.; Jia, Y. Rediscovering black phosphorous as an anisotropic layered material for optoelectronics and electronics. Nat. Commun. 2014, 5, 448.

12. Roldán, R.; Chirolli, L.; Prada, E.; Silva-Guillén, J. A.; San-Jose, P.; Guinea, F. Theory of 2D crystals: graphene and beyond. Chem. Soc. Rev. 2017, 46, 4387-4399.

13. Qiao, J.; Kong, X.; Hu, Z.-X.; Yang, F.; Ji, W. High-mobility transport anisotropy and linear dichroism in few-layer black phosphorous. Nat. Commun. 2014, 5, 4475.

14. Island, J. O.; Buscema, B.; Barawi, M.; Clamagirand, J. M.; Ares, J. R.; Sánchez, C.; Ferrer, I. J.; Steele, G. A.; van der Zant, H. S.; Castellanos-Gomez, A. Ultrahigh photoresponseof few-layer $\mathrm{TiS}_{3}$ nanoribbon transistors. Adv. Opt. Mater. 2014, 2, 641-645.

15. Island, J. O.; Barawi, M.; Biele, R.; Almazán, A.; Clamagirand, J. M.; Ares, J. R.; Sánchez, C.; van der Zant, H. S. J.; Álvarez, J. V.; D’Agosta, R. et al. TiS 3 Transistors with Tailored Morphology and Electrical Properties. Adv. Mater. 2015, 27, 2595-2601.

16. Lipatov, A.; Wilson, P. M.; Shekhirev, M.; Teeter, J. D.; Netusil, R.; Sinitskii, A. Few-layered titanium trisulfide $\left(\mathrm{TiS}_{3}\right)$ field-effect transistors. Nanoscale 2015, 7, 1229112296.

17. Osada, K.; Bae, S.; Tanaka, M.; Raebiger, H.; Shudo, K.; Suzuki, T. Phonon frequencies of few-layer crystals of quasi-one-dimensional $\mathrm{ZrS}_{3}$ and $\mathrm{ZrSe}_{3}$. J. Phys. Chem. C 2016, $120,4653-4659$. 
18. Jin, Y.; Li, X.; Yang, J. Single layer of $\mathrm{MX}_{3}(\mathrm{M}=\mathrm{Ti}, \mathrm{Zr} ; \mathrm{X}=\mathrm{S}$, Se, Te): a new platform for nano-electronics and optics. Phys. Chem. Chem. Phys. 2015, 17, 18665-18669.

19. Dai, J.; Zeng, X. C. Titanium Trisulfide Monolayer: Theoretical Prediction of a New Direct-Gap Semiconductor with High and Anisotropic Carrier Mobility. Angew. Chem. Int. Ed. 2015, 54, 7572-7576.

20. Li, M.; Dai, J.; Zeng, X. C. Tuning the electronic properties of transition-metal trichalcogenides via tensile strain. Nanoscale 2015, 7, 15385-15391.

21. Iyikanat, F.; Sahin, H.; Senger, R. T.; Peeters, F. M. Vacancy formation and oxidation characteristics of single later $\mathrm{TiS}_{3}$. J. Phys. Chem. C 2015, 119, 10709-10715.

22. Dai, J.; Li, M.; Zeng, X. C. Group IVB transition metal trichalcogenides: a new class of 2D layered materials beyond graphene. WIREs Computational Molecular Science 2016, $6,211-222$.

23. Silva-Guillén, J. A.; Canadell, E.; Ordejón, P.; Guinea, F.; Roldán, R. Anisotropic features in the electronic structure of the two-dimensional transition metal trichalcogenide $\mathrm{TiS}_{3} 3$ : electron doping and plasmons. 2D Materials 2017, 4, 025085.

24. Pant, A.; Torun, E.; Chen, B.; Bhat, S.; Fan, X.; Wu, K.; Wright, D. P.; Peeters, F. M.; Soignard, E.; Sahin, H. et al. Strong dichroic emission in the pseudo one dimensional material $\mathrm{ZrS}_{3}$. Nanoscale 2016, 8, 16259-16265.

25. Aierken, Y.; Çakir, D.; Peeters, F. M. Strain enhancement of acoustic phonon limited mobility in monolayer $\mathrm{TiS}_{3}$. Phys. Chem. Chem. Phys. 2016, 18, 14434-14441.

26. Kang, J.; Sahin, H.; Peeters, F. M. Mechanical properties of monolayer sulphides: a comparative study between $\mathrm{MoS}_{2}, \mathrm{HfS}_{2}$ and $\mathrm{TiS}_{3}$. Phys.Chem.Chem. Phys. 2017, 17, $27742-27749$. 
27. Wu, K.; Torun, E.; Sahin, H.; Chen, B.; Fan, X.; Pant, A.; Parsons Wright, D.; Aoki, T.; Peeters, F. M.; Soignard, E. et al. Unusual lattice vibration characteristics in whiskers of the pseudo-one-dimensional titanium trisulfide $\mathrm{TiS}_{3}$. Nanoscale 2016, 7, 12952.

28. Kong, W.; Bakaksiz, C.; Chen, B.; Wu, K.; Blei, M.; Fan, X.; Shen, Y.; Sahin, H.; Wright, D.; Narang, D. S. et al. Angle resolved vibrational properties of anisotropic transition metal trichalcogenide nanosheets. Nanoscale 2017, 9, 4175-4182.

29. Stauber, T. Plasmonics in Dirac systems: from graphene to topological insulators. $J$. Phys.: Condens. Matter 2014, 26, 123201.

30. Grigorenko, A. N.; Polini, M.; Novoselov, K. S. Graphene plasmonics. Nat. Photonics 2012, 6, 749-758.

31. Low, T.; Roldán, R.; Wang, H.; Xia, F.; Avouris, P.; Moreno, L. M.; Guinea, F. Plasmons and Screening in Monolayer and Multilayer Black Phosphorus. Phys. Rev. Lett. 2014, $113,106802$.

32. Liu, Z.; Aydin, K. Localized surface plasmons in nanostructured monolayer black phosphorus. Nano Lett. 2016, 16, 3457-3462.

33. Nemilentsau, A.; Low, T.; Hanson, G. Anisotropic 2D Materials for Tunable Hyperbolic Plasmonics. Phys. Rev. Lett. 2016, 116, 066804.

34. San-Jose, P.; Parente, V.; Guinea, F.; Roldán, R.; Prada, E. Inverse Funnel Effect of Excitons in Strained Black Phosphorus. Phys. Rev. X 2016, 6, 031046.

35. Fei, R.; Faghaninia, A.; Soklaski, R.; Yan, J.-A.; Lo, C.; Yang, L. Enhanced thermoelectric efficiency via orthogonal electrical and thermal conductances in phosphorene. Nano Lett. 2014, 14, 6393-6399.

36. Yi, H.; Komesu, T.; Gilbert, S.; Hao, G.; Yost, A. Y.; Lipatov, A.; Sinitskii, A.; Avila, J.; 
Chen, C.; Asensio, M. C. et al. The band structure of the quasi-one-dimensional layered semiconductor $\mathrm{TiS}_{3}(001)$. Appl. Phys. Lett. 2018, 112, 052102.

37. Kang, K.; Wang, L.-W. Robust band gap of TiSe 3 nanofilms. Phys. Chem. Chem. Phys. 2016, 18, 14805-14809.

38. Heyd, J.; Scuseria, G. E.; Ernzerhof, M. Erratum: Hybrid functionals based on a screened Coulomb potential [J. Chem. Phys. 118, 8207 (2003)]. The Journal of Chemical Physics 2006, 124, 219906.

39. Biele, R.; Flores, E.; Ares, J. R.; Sánchez, C.; Ferrer, I. J.; Rubio-Bollinger, G.; Castellanos-Gomez, A.; D’Agosta, R. Strain-induced band gap engineering in layered TiS3. Nano Research 2018, 11, 225-232.

40. Sevik, C.; Wallbank, J. R.; Gülseren, O.; Peeters, F. M.; Çakır, D. Gate induced monolayer behavior in twisted bilayer black phosphorus. 2D Mater. 2017, 4, 035025.

41. Island, J. O.; Biele, R.; Barawi, M.; Clamagirand, J. M.; Ares, J. R.; Sánchez, C.; Van Der Zant, H. S.; Ferrer, I. J.; D’Agosta, R.; Castellanos-Gomez, A. Titanium trisulfide (TiS 3): a 2D semiconductor with quasi-1D optical and electronic properties. Scientific reports 2016, 6, 22214.

42. Sakuma, T.; Nishino, S.; Miyata, M.; Koyano, M. Thermoelectric Properties for a Suspended Microribbon of Quasi-One-Dimensional $\mathrm{TiS}_{3}$. Journal of Electronic Materials 2018, 47,3177 .

43. Low, T.; Chaves, A.; Caldwell, J. D.; Kumar, A.; Fang, N. X.; Avouris, P.; Heinz, T. F.; Guinea, F.; Martin-Moreno, L.; Koppens, F. Polaritons in layered 2D materials. Nature Mat. 2017, 16, 182-194.

44. Hohenberg, P.; Kohn, W. Inhomogeneous Electron Gas. Physical Review 1964, 136, B864. 
45. Kohn, W.; Sham, L. J. Self-Consistent Equations Including Exchange and Correlation Effects. Physical Review 1965, 140, A1133.

46. Soler, J. M.; Artacho, E.; Gale, J. D.; García, A.; Junquera, J.; Ordejón, P.; SánchezPortal, D. The SiEsTA method for ab initio order-N materials simulation. Journal of Physics: Condensed Matter 2002, 14, 2745.

47. Artacho, E.; Anglada, E.; Diéguez, O.; Gale, J. D.; García, A.; Junquera, J.; Martin, R. M.; Ordejón, P.; Pruneda, J. M.; Sánchez-Portal, D. et al. The Siesta method; developments and applicability. Journal of Physics: Condensed Matter 2008, 20, 064208.

48. Perdew, J. P.; Burke, K.; Ernzerhof, M. Generalized Gradient Approximation Made Simple. Physical Review Letters 1996, 77, 3865.

49. Troullier, N.; Martins, J. L. Efficient pseudopotentials for plane-wave calculations. Physical Review B 1991, 43, 1993.

50. Kleinman, L.; Bylander, D. M. Efficacious Form for Model Pseudopotentials. Physical Review Letters 1982, 48, 1425.

51. Louie, S. G.; Froyen, S.; Cohen, M. L. Nonlinear ionic pseudopotentials in spin-densityfunctional calculations. Physical Review B 1982, 26, 1738.

52. Artacho, E.; Sánchez-Portal, D.; Ordejón, P.; García, A.; Soler, J. M. Linear-Scaling ab-initio Calculations for Large and Complex Systems. Physica Status Solidi (b) 1999, 215, 809 .

53. Monkhorst, H. J.; Pack, J. D. Special points for Brillouin-zone integrations. Physical Review B 1976, 13, 5188.

54. Ando, T.; Fowler, A. B.; Stern, F. Electronic properties of two-dimensional systems. Rev. Mod. Phys. 1982, 54, 437-672. 
55. Giuliani, G. F.; Vignale, G. Quatum Theory of the Electron Liquid; CUP, Cambridge, 2005. 
Graphical TOC Entry

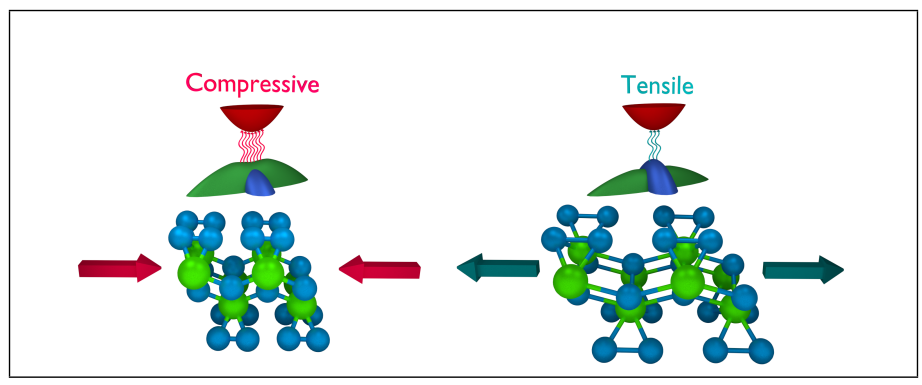

